\title{
ASSESSING THE RELIABILITY AND THE ACCURACY OF ATTITUDE EXTRACTED FROM VISUAL ODOMETRY FOR LIDAR DATA GEOREFERENCING
}

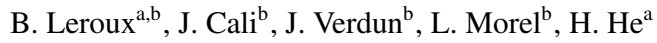 \\ ${ }^{a}$ Hélicéo - Geomatic Innovation and Technology, 6 rue Rose Dieng-Kuntz 44300 Nantes, France - (boris.leroux, haixing.he)@ heliceo.fr \\ ${ }^{\mathrm{b}}$ Geomatics and Land Law Lab (GeF/L2G/Cnam EA 4630) - ESGT - 1 Bd Pythagore, 72000 Le Mans, France - \\ (jose.cali, jerome.verdun, laurent.morel)@lecnam.net
}

\section{Commission VI, WG VI/4}

KEY WORDS: LiDAR, Visual Odometry, UAV, Direct Georeferencing

\begin{abstract}
:
Airborne LiDAR systems require the use of Direct Georeferencing (DG) in order to compute the coordinates of the surveyed point in the mapping frame. An UAV platform does not derogate to this need, but its payload has to be lighter than this installed onboard so the manufacturer needs to find an alternative to heavy sensors and navigation systems. For the georeferencing of these data, a possible solution could be to replace the Inertial Measurement Unit (IMU) by a camera and record the optical flow. The different frames would then be processed thanks to photogrammetry so as to extract the External Orientation Parameters (EOP) and, therefore, the path of the camera. The major advantages of this method called Visual Odometry (VO) is low cost, no drifts IMU-induced, option for the use of Ground Control Points (GCPs) such as on airborne photogrammetry surveys. In this paper we shall present a test bench designed to assess the reliability and accuracy of the attitude estimated from VO outputs. The test bench consists of a trolley which embeds a GNSS receiver, an IMU sensor and a camera. The LiDAR is replaced by a tacheometer in order to survey the control points already known. We have also developped a methodology applied to this test bench for the calibration of the external parameters and the computation of the surveyed point coordinates. Several tests have revealed a difference about 2-3 centimeters between the control point coordinates measured and those already known.
\end{abstract}

\section{INTRODUCTION}

\subsection{Context}

The surveying sector is still looking for denser, faster and more accurate data collection. The recent development of professional UAV and lightweight embedded systems has allowed to design new platforms that will meet this demand. The use of UAVs with photogrammetry onboard has proved his value for global mapping applications but it requires a long post-processing time and is unefficient for objects with complex geometry and for homogeneous surfaces. The LiDAR (Light Detection and Ranging) systems, which measure ranges and angles, have proved valuable for a lot of applications such as: power line detection and inspection [Deng et al., 2014], forest inventory [Wallace et al., 2012] or topographic mapping [Lin et al., 2011]. Therefore, we intend to design a LiDAR UAV platform to meet the surveying demand and to supply the photogrammetry limits.

Compared to aerial photogrammetry where the point clouds can be georeferenced by Ground Control Points (GCPs), LiDAR data need Direct Georeferencing (DG). In order to compute the target coordinates, we need to know the exact position and attitude of the sensor at the time it was shot. DG generally requires both a Global Navigation Satellite System (GNSS) receiver for measuring the position and Attitude and Heading Reference System (AHRS) for measuring the attitude, both with sufficient accuracy - that is a centimeter accuracy for position and 0.01 degree accuracy for attitude - [Skaloud et al., 1996].

Note: While the acronym IMU designates a platform that consists of 3 accelerometers and 3 gyrometers, the acronym AHRS refers to the sensor which contains an IMU and a computer so as to provide attitude measurements. As for the Inertial Navigation System (INS), it designates a navigation aid based on an IMU, that can calculate the position, velocity and attitude of both manned and unmanned vehicles.

\subsection{Principle of direct georeferencing}

The DG concept consists in computing the coordinates of a surveyed point in the mapping frame (Fig. 1) $\Upsilon_{m a p}$ using GNSS/IMU outputs. Mathematical notations are the following:

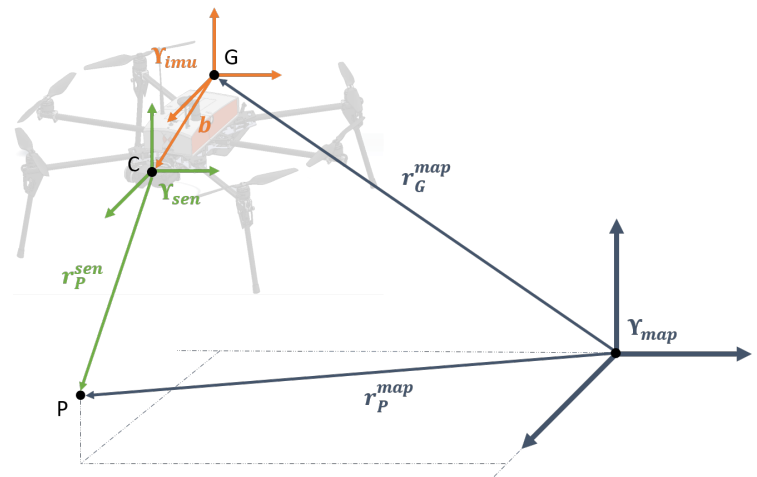

Figure 1. Principle of direct georeferencing - definition of reference frames

with:

- $P$ : Surveyed point;

- $G$ : Phase center of the GNSS receiver antenna; 
- $C$ : Sensor center of acquisition;

- $\Upsilon_{m a p}$ : Mapping frame;

- $\Upsilon_{i m u}$ : IMU frame. The origin of this frame is the point $G$;

- $\Upsilon_{\text {sen }}$ : Sensor frame. The origin of this frame is the point $C$;

- $\mathbf{r}_{A}^{f}$ : Vector the components of which correspond to the point $A$ coordinates in the frame $f$;

- b: Lever-arm vector which corresponds to the spatial offset between the antenna phase center and sensor frame origin.

The coordinates of point $P$ can be computed with the following mathematical expression (1):

$$
\mathbf{r}_{P}^{\text {map }}=\mathbf{r}_{G}^{\text {map }}+R_{\text {nav }}^{\text {map }} R_{i m u}^{\text {nav }} R_{\text {sen }}^{i m u}\left(b+\mathbf{r}_{P}^{\text {sen }}\right)
$$

where:

- $R_{\text {nav }}^{\text {map }}$ : Transformation matrix between $\Upsilon_{n a v}$ and $\Upsilon_{\text {map }}$ reference frames. The navigation frame is a local geographic frame the origin of which is at the location of the INS. Its 3 axes are aligned with the directions of East (X), North (Y) and the normal to an Earth-centered rotational ellipsoid oriented upward (Z). The transformation matrix depends on the vehicle position as follows:

$$
R_{\text {map }}^{\text {nav }}=R_{Z}\left(\lambda+\frac{\pi}{2}\right) R_{X}\left(\frac{\pi}{2}-\psi\right)
$$

where $\psi$ and $\lambda$ denote respectively the vehicle latitude and longitude, $R_{X}$ and $R_{Z}$ the rotation matrices around respectively the axes $\mathrm{X}$ and $\mathrm{Z}$.

- $R_{i m u}^{n a v}$ : Transformation matrix between $\Upsilon_{n a v}$ and $\Upsilon_{i m u}$ reference frames. The matrix values can be calculated from the vehicle attitude angles - that is the roll (r), pitch (p) and yaw $(y)$ - by means of the following mathematical expression:

$$
R_{i m u}^{n a v}=R_{Z}(y) R_{Y}(p) R_{X}(r)
$$

It should be noted that the IMU reference frame and equation 3 depend on the IMU model. We have therefore used a standard convention.

- $R_{s e n}^{i m u}$ : Transformation matrix between $\Upsilon_{s e n}$ and $\Upsilon_{i m u}$ reference frames. This matrix is commonly named boresight matrix. The boresight matrix differs from the identity matrix as soon as the IMU and sensor frames are misaligned.

The lever-arm vector and boresight matrix form the External Parameters (EP). They are generally calculated during a dedicated calibration flight over an aera with GCPs.

As mentioned before, DG generally requires high grade IMUs which are generally heavy and expensive, and thus not compatible with UAVs. Some integrated lightweight Original Equipment Manufacturers (OEMs) propose a combined system GNSS+IMU with a sufficient accuracy such as Applanix [Applanix, n.d.], but it costs of several ten of thousands euros makes it not affordable for small platforms. Another drawback with IMU is the drift, which can degrade dramatically the accuracy of the system [El-Sheimy, 2009].
DG on UAV has taken advantage of the miniaturization of INS and the development of digital photogrammetry. For example [Daakir et al., 2015] develops a processing method suitable for data acquired on a light UAV. The system can reach a very high positioning accuracy (down to $1 \mathrm{~cm}$ ) on check points using only one GCP. [Eling et al., 2015] also presents a system which can reach a high accuracy $(3-5 \mathrm{~cm})$ for a survey without the prerequired GCP procedure. These authors suggest that DG can be carried out on UAV with photogrammetry onboard for topographic applications.

Today DG of LiDAR UAV platforms is not as advanced as for photogrammetry UAV platforms. Among the available DG solutions for photogrammetry, we can find development versions such as those described in [Wallace et al., 2012] and [Lin et al., 2011], as well as commercial versions such as Rigel Ricopter. Manufacturers also focus on the development of "POD" solutions, which consist of a LiDAR sensor and a GNSS/IMU OEM, easily integrated on existing UAV platforms. As an illustration of companies which propose such solutions, we can cite YellowScan, RouteScene and Phoenix Aerial. Sofar the DG of LiDAR data has required GNSS/INS to measure the attitude angles of the carrier vehicle. As mentioned before, the cost and weight of current GNSS/INS systems is not well-suited to light UAVs.

An alternative to INS could be a camera capturing pictures or recording a video during the flight. The trajectory of the sensor would then be directly extracted from the picture frames thanks to image matching. This method is called Visual Odometry (VO).

\subsection{Principle of Visual Odometry}

The problem is to determine the transformation between successive picture frames from their content. Several algorithms exist, but the principal steps can be summarized as follows:

1. Keypoints detection: There are many keypoint detectors but the most famous is Scale Invariant Feature Transform (SIFT) from [Lowe, 2004]. It makes use of high contrast regions inside the images. Each keypoint is then affected with a descriptor (128 dimensions) that describes locally the orientations of the image contrast gradients.

2. Image matching: Keypoint descriptors of the different images are compared to find matches. For each image, SIFT can compute about 1000 keypoints, which can then be compared together. Any keypoint having the same descriptor whatever the image it belongs to, becomes a tie point.

3. Pose estimate: With sufficient tie points, it is possible to compute the External Orientation Parameters (EOP) of the camera for each picture frame. They correspond to 6 parameters - that is the camera center $\left(S_{X}, S_{Y}, S_{Z}\right)$ and the 3D rotation angles $(\omega, \phi, \kappa)$ - Usually one picture frame is fixed as reference, and thus its EOP are set to 0 or specific values.

We have therein used digital photogrammetry softwares so as to applied SIFT on image datasets and appraise EOPs.

VO is more famous for its application in the robotic field, especially in navigation and localization. For example, the space mission on Mars that started in 2003 [Maimone et al., 2007] has involved two rovers making use of VO to explore Mars surface and 
geology. Another trendy field in VO research is autonomous vehicles. For example [Howard, 2008] describes a visual odometry algorithm to estimate frame-to-frame camera motion from successive stereo image pairs. VO systems are also used for UAVs navigation for instance in environments with lack of GNSS satellite coverage [Caballero et al., 2008]. As described here, VO is already used for navigation and localization but its use for DG is not common. Now lightweight and low-cost sensors which provide high rate and high resolution frames, have become available. This optical information could be useful for attitude determination. The disadvantage of VO compared to AHRS is the post-processing or the required computing ressources for realtime processing.

As mentioned before, we intend to design a LiDAR UAV platform which takes advantage of VO for attitude determination. In this paper, we shall present an experimental mobile test bench used to assess the reliability and the accuracy of visual odometry in order to georeference LiDAR data. The test bench is equipped with different instruments each one simulating a particular component of the future LiDAR UAV platform. The second part will focus on the DG methodology carried out with our test bench, particularly how the EPs have been adjusted and how the surveyed points have been georeferenced. The third part will describe the equipment, the testing field, the protocol and data processing. The fourth part discusses the results of two measurement campaigns we carried out. The conclusions and prospects of our work are delineated in the fifth part. This project has made use of the techniques in the fields of dynamic positioning and moving gravimetry [Roussel et al., 2015] already validated by the Geomatics and Land Law Laboratory (GeF/L2G) based in Le Mans (France).

\section{METHODOLOGY}

In this section, the theoretical concepts of our methodology will be carefully detailed. First, we have transposed the principle of DG to our own mobile test bench, for which the LiDAR data are assumed to be acquired by a tacheometer and the IMU data are replaced by the pictures provided by a camera. By doing so, the sensor reference frame $\Upsilon_{\text {sen }}$ has to be replaced by the tacheometer frame $\Upsilon_{t}$ and the IMU reference frame $\Upsilon_{i m u}$ is replaced by the camera reference frame $\Upsilon_{\text {cam }}$ (Fig. 2). According to the

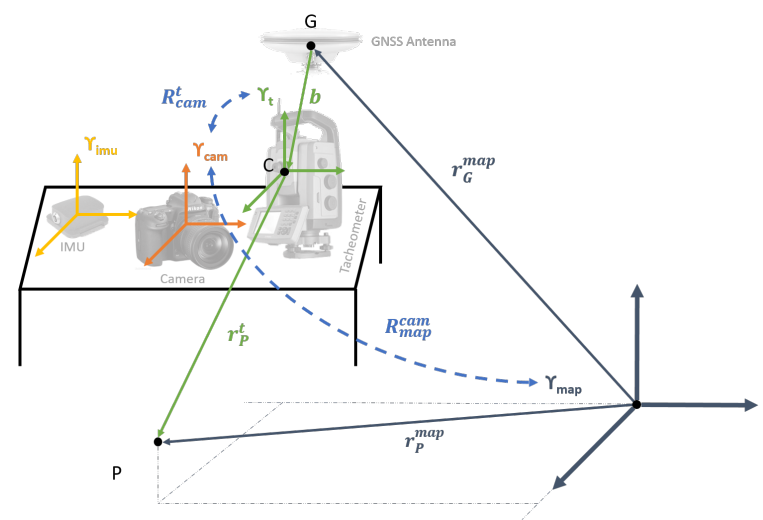

Figure 2. Definition of reference frames for our own test bench.

figure 2, the DG equation (1) can be rewritten for our case as:

$$
\mathbf{r}_{P}^{\text {map }}=\mathbf{r}_{G}^{\text {map }}+R_{c a m}^{\text {map }} R_{t}^{c a m}\left(\mathbf{r}_{P}^{t}+\mathbf{b}\right)
$$

where:
- $\mathbf{r}_{P}^{\text {map }}:$ is a vector the component of which are the coordinates of point $P$ in the mapping frame;

- $\mathbf{r}_{G}^{\text {map }}:$ is the position vector whose components are the coordinates of point $G$ measured by the GNSS receiver;

- $R_{c a m}^{\text {map }}$ : is the transformation matrix from the camera frame to the mapping frame. This matrix is derived from the EOP angles $(\omega, \phi, \kappa)$ computed by means of a photogrammetry software. The values of the matrix depend on the convention adopted in the post-processing. In our case, we have used the PATB convention [Bäumker and Heimes, 2001] of Pix4D software (Section. 3.4). The $R_{c a m}^{m a p}$ is then given by:

$$
R_{\text {cam }}^{\text {map }}=R_{X}(\omega) R_{Y}(\phi) R_{Z}(\kappa)
$$

It should be noted that the transformation from $\Upsilon_{\text {map }}$ to $\Upsilon_{n a v}$ reference frames is unecessary because the photogrammetryprovided EOPs are already expressed in $\Upsilon_{m a p}$;

- $R_{t}^{\text {cam }}:$ is the transformation matrix between $\Upsilon_{t}$ to $\Upsilon_{\text {cam }}$ reference frames, that is the boresight matrix;

- $\mathbf{r}_{P}^{t}$ : is the vector whose components are the coordinates of point $P$ in the tacheometer frame. The coordinates are computed from the tacheometer measurements - that is the range $(d)$, the horizontal direction angle $\left(H_{Z}\right)$ and vertical direction angle $\left(V_{Z}\right)$ as follows:

$$
\left(\begin{array}{c}
X \\
Y \\
Z
\end{array}\right)=\left(\begin{array}{c}
d \sin \left(H_{z}\right) \sin \left(V_{z}\right) \\
d \cos \left(H_{z}\right) \sin \left(V_{z}\right) \\
d \cos \left(V_{z}\right)
\end{array}\right)
$$

- b: is the lever-arm between the GNSS antenna phase center and the origin of the tacheometer frame.

Prior to any measurement with our test bench, this one has to be adjusted by determining the EPs, that is the lever-arm vector components and the values of the boresight matrix. To perform this adjustment, the EPs are derived from the differences between measured coordinates and accurate coordinates of calibration points, visible as colored markings on a wall (Fig. 5). The problem is tantamount to estimate the rigid body ransformation parameters between two reference frames $\Upsilon_{1}$ and $\Upsilon_{2}$ (Fig. 3), which can be done by means of the Singular Value Decomposition (SVD) [Challis, 1995]. For this kind of transformation the parameters to be estimated are:

- $R_{\Upsilon_{2}}^{\Upsilon_{1}}$ : Transformation matrix between $\Upsilon_{1}$ and $\Upsilon_{2}$, which corresponds to a 3D rotation;

- $T_{\Upsilon_{2}}^{\Upsilon_{1}}$ : Translation vector between $\Upsilon_{1}$ and $\Upsilon_{2}$.

Given the vectors $\mathbf{x}_{i}^{\Upsilon_{1}}$ et $\mathbf{x}_{i}^{\Upsilon_{2}}$ of the coordinates of $\mathrm{n}$ points known in both reference frames, the parameters can be determined by minimizing the energy function $\Sigma$ defined as:

$$
\Sigma=\sum_{i=1}^{n}\left\|\mathbf{x}_{i}^{\Upsilon_{1}}-R_{\Upsilon_{2}}^{\hat{\Upsilon}_{1}} \mathbf{x}_{i}^{\Upsilon_{2}}-T_{\Upsilon_{2}}^{\hat{\Upsilon}_{1}}\right\|^{2}
$$

Assuming rigid body transformation, the distance between the points and their centroid is the same for both $\Upsilon_{1}$ and $\Upsilon_{2}$ frames. Given the following quantities: 


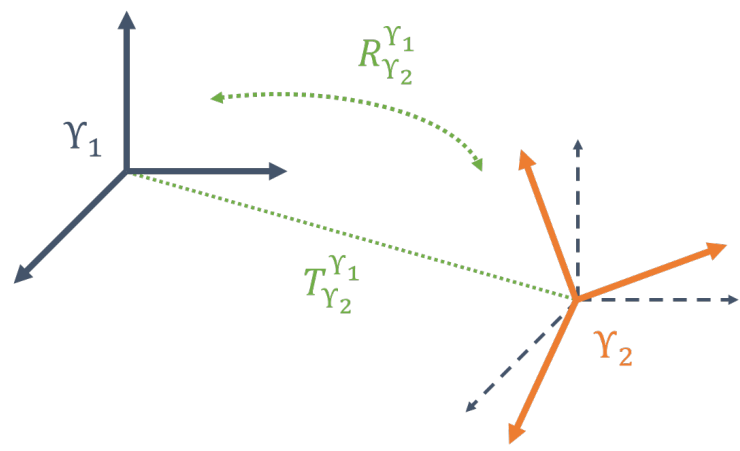

Figure 3. Rigid body transformation parameters between two arbitrary reference frames

- $\mathbf{x}_{B}^{\Upsilon_{1}}$ : Coordinates of the centroid of points $i=1,2, \ldots, n$ in $\Upsilon_{1}$.

- $\mathbf{x}_{B}^{\Upsilon_{2}}$ : Coordinates of the centroid of points $i=1,2, \ldots, n$ in $\Upsilon_{2}$.

We can then write:

$$
T_{\Upsilon_{2}}^{\hat{\Upsilon}_{1}}=\mathbf{x}_{B}^{\Upsilon_{1}}-R_{\Upsilon_{2}}^{\hat{\Upsilon}_{1}} \mathbf{x}_{B}^{\Upsilon_{2}}
$$

By replacing this term in equation (7), we obtain an equation of energy involving solely the rotation matrix $R_{\Upsilon_{2}}^{\Upsilon_{1}}$ :

$$
\Sigma=\sum_{i=1}^{n}\left\|\left(\mathbf{x}_{i}^{\Upsilon_{1}}-\mathbf{x}_{B}^{\Upsilon_{1}}\right)-R_{\Upsilon_{2}}^{\hat{\Upsilon}_{1}}\left(\mathbf{x}_{i}^{\Upsilon_{2}}-\mathbf{x}_{B}^{\Upsilon_{2}}\right)\right\|^{2}
$$

By decomposing the $3 \times 3$ correlation matrix $H$ defined by:

$$
H=\sum_{i=1}^{n}\left(\mathbf{x}_{i}^{\Upsilon_{1}}-\mathbf{x}_{B}^{\Upsilon_{1}}\right)\left(\mathbf{x}_{i}^{\Upsilon_{2}}-\mathbf{x}_{B}^{\Upsilon_{2}}\right)^{T}
$$

by means of SVD, we get:

$$
H=U S V^{T}
$$

Then the energy $\Sigma$ is minimal when:

$$
R_{\Upsilon_{2}}^{\hat{\Upsilon}_{1}}=V U^{T}
$$

Once $R_{\Upsilon_{2}}^{\hat{\Upsilon}_{1}}$ is determined, $T_{\Upsilon_{2}}^{\hat{\Upsilon}_{1}}$ can be retrieved from equation (8). In our case we have evaluated $R_{t}^{\text {map }}$ and $\mathbf{r}_{C}^{\text {map }}$ with 3 known points in both the mapping frame $\left(\Upsilon_{\text {map }}\right)$ and the tacheometer frame $\left(\Upsilon_{t}\right)$. The EPs can then be calculated by means of the following relationships:

$$
\begin{gathered}
\mathbf{b}=\left(R_{t}^{\text {map }}\right)^{-1}\left(\mathbf{r}_{\text {map }}^{C}-\mathbf{r}_{\text {map }}^{G}\right) \\
R_{t}^{c a m}=\left(R_{m a p}^{\text {cam }}\right) R_{t}^{\text {map }}
\end{gathered}
$$

In the survey phase, we have made use of the equation 4 with the EPs so as to compute the coordinates of the points surveyed by the platform.

\section{EXPERIMENTS}

Our mobile test bench consists of several instruments described subsequently. Such a test bench allows photogrammetry and laser scanning surveys in a stop-and-go mode to be carried out. The overall quality of the data acquired with the test bench can be assessed by comparing the measured coordinates of control points found in the surveyed scene to their accurate coordinates already known.

\subsection{Equipments}

Several devices are mounted on a trolley that forms the test bench (Fig. 4):

- Tacheometer: like LiDAR sensors, this instrument is also a range scanning system, but, contrary to LiDAR that acquired cloud points, it allows to survey individual points in the scene with high accuracy. We can therefore focus on the control points involved in the assessment of the test bench.

- Camera: we have employed a standard digital camera, ensuring that the same settings and focal length are constant during experiments.

- GNSS receiver: to measure the position of the trolley.

- IMU: we have also utilized an AHRS, MTI XSens to compare the results with those computed with VO.

The trolley can be easily moved from one station to the other.

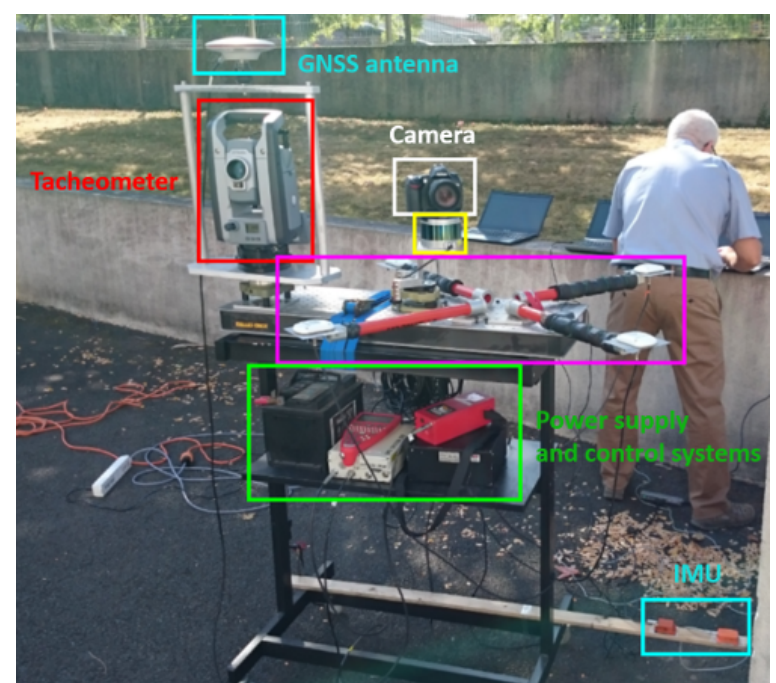

Figure 4. View of the test bench.

\subsection{Test Field}

The test field is divided in two scenes:

- The first one is a wall equipped with targets (Fig. 5), forming a calibration polygon whose vertices are the control points. Coordinates of the control points have been determined by classical topography methods and expressed in the French national reference frame RGF93. The overall accuracy of these coordinates is of $3 \mathrm{~cm}$. It should be noticed that this value results not only from measurement errors, but also from georeferencing errors. This scene has been used for the adjustment phase.

- The second one is a set of houses also equipped with targets (Fig. 6), which will be called, from now on, house set. As for the first scene, the coordinates of control points are known with $3 \mathrm{~cm}$ accuracy. This set has been used for the calibration phase. 


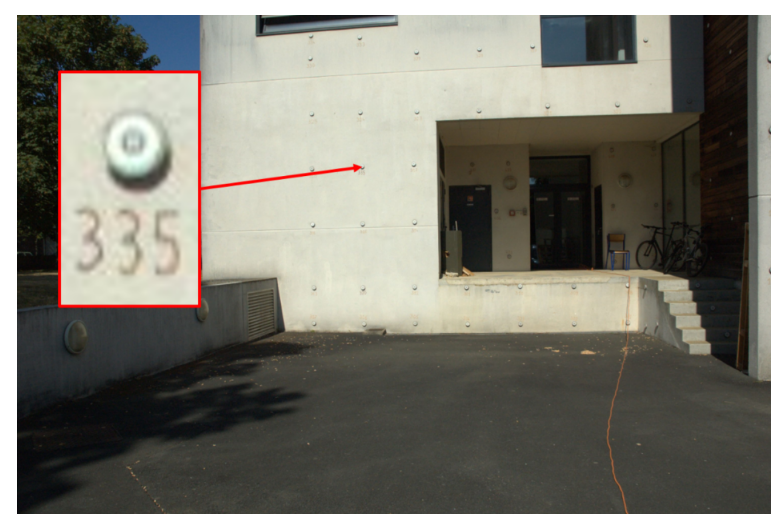

Figure 5. Calibration polygon

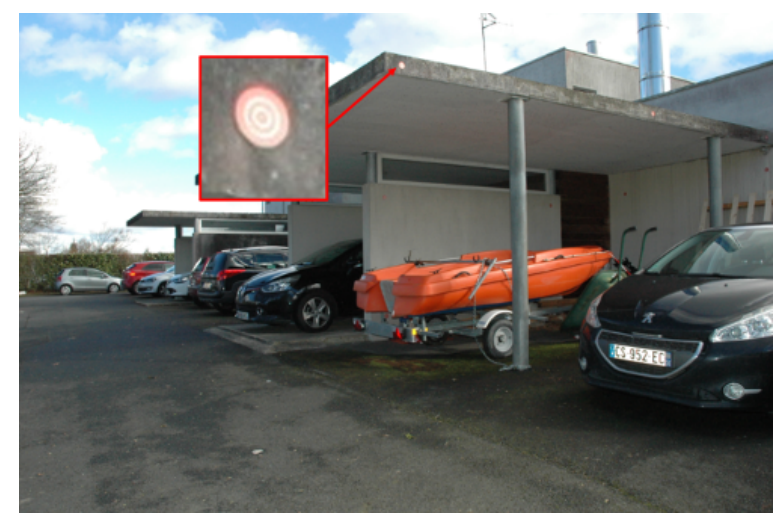

Figure 6. House set

\subsection{Measurement and Calibration Protocol}

The protocol of our experiments can be divided into two phases:

- The adjustment phase which consists in determining the exterior parameters of our test bench;

- The calibration phase which consists in collecting measurements with all sensors and to control the accuracy using the control points.

During both phases, we have repeated the same operation, changing only the scenes. Several stations have been occupied. The canvas of the stations is designed to look like an aerial photogrammetric survey canvas, including two flight axes and an overlap between images (Fig. 7). For each station, the steps of the survey have been the following:

- At least, 3 targets have been surveyed with the tacheometer;

- The position of the test bench at the station has been measured with the GNSS receiver;

- The attitude of the test bench at the station has been measured with the AHRS;

- One image per station has been captured with the camera.

For the adjustment phase, 8 stations have been occupied with the test bench oriented along 2 different axes ( 5 stations for the first axis, 3 stations for the second one). All the images and the

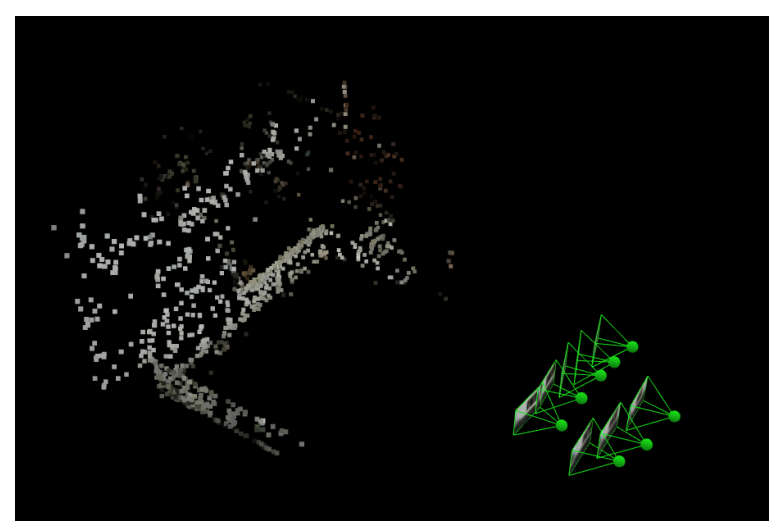

Figure 7. Image canvas for adjustment phase

tacheometer measurements have been headed towards the calibration polygon (Fig. 7). During the phase, the same 5 targets have been surveyed.

For the calibration phase, 12 stations have been occupied, 8 with the test bench oriented along the first axis, and 4 oriented along the second one. All the images and the tacheometer measurements have been headed towards the house set (Fig. 8). All the available targets have been used this time in order to gain a maximum of control points.

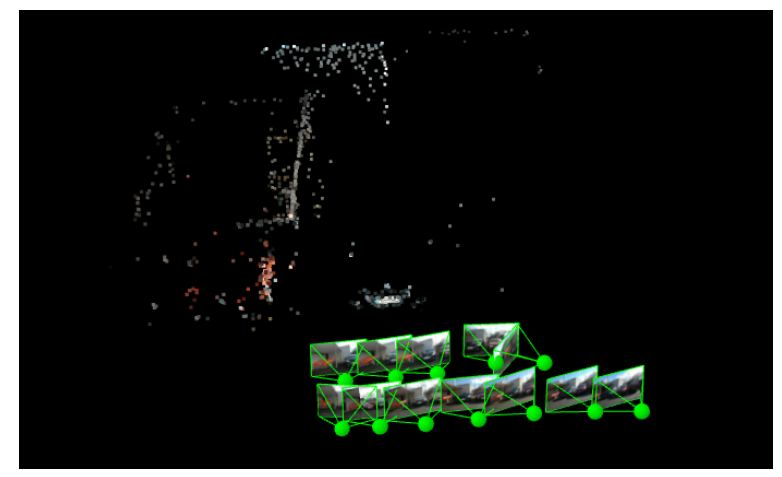

Figure 8. Image canvas for the calibration phase

\subsection{Post-processing}

The post-processing has been the same for both phases but depends on the sensor:

- Tacheometer: For each station, the Cartesian coordinates of each target have been calculated from the range, the horizontal and vertical direction angles acquired by the tacheometer (Eq. 6).

- GNSS: the RINEX files have been post-processed using the Permanent GNSS Network from France (RGP) and particularly the station MAN2 which is located on the top of the calibration polygon. The baselines are therefore very short. The post-processing has been performed with Leica GeoOffice software.

- AHRS: For each station, attitude measurements have been performed during at least 1 minute at a frequency sampling of $20 \mathrm{~Hz}$, such giving time series of attitude angles. The resulting attitude angles, that is roll, pitch and yaw, have been deduced from the time series by averaging the acquired values. 
- Images: we have split the two phases because there is no correspondence between the images. The images of both phases have then been processed with Pix4D software. From the software, we have got the EOPs of each image. At this stage, the images have been georeferenced using the targets as GCPs (Section. 5).

Finally, for each station we have computed:

- The position of the antenna phase center in the mapping frame;

- The attitude angles given by the AHRS;

- The EOPs of the camera;

- The Cartesian coordinates of the targets surveyed at each station in the tacheometer frame.

\section{RESULTS}

Several experiments have been performed with the test bench, but the paper will focus on the most recent carried out in February 2017.

\subsection{Adjustment results}

As explained in section 2, a set of EPs has been adjusted. Table 1 shows the values obtained for the lever-arm. Table 2 and 3 shows the angular eccentricities deduced from the rotation matrix $R_{t}^{\text {map }}$ using the following relation:

$$
\begin{aligned}
& e_{X}=c \operatorname{Arcsin}\left(m_{1,3}\right) \\
& e_{Y}=\operatorname{Arctan}\left(-m_{2,3} / m_{3,3}\right) \\
& e_{Z}=\operatorname{Arctan}\left(-m_{1,2} / m_{1,1}\right)
\end{aligned}
$$

where $m_{i, j}$ with $(i, j) \in \llbracket 1,3 \rrbracket$ correspond to values of $R_{t}^{\text {map }}$. Note: this relation is used only for eccentricities adjusted from VO.

\begin{tabular}{c|c|c|c} 
Station & $b_{X}[\mathrm{~m}]$ & $b_{Y}[\mathrm{~m}]$ & $b_{Z}[\mathrm{~m}]$ \\
\hline S1 & -0.0233 & -0.005 & -0.22 \\
\hline S2 & -0.011 & 0.008 & -0.221 \\
\hline S3 & -0.014 & 0.004 & -0.237 \\
\hline S4 & -0.01 & -0.002 & -0.227 \\
\hline S5 & -0.011 & 0.003 & -0.226 \\
\hline S6 & -0.013 & -0.006 & -0.228 \\
\hline S7 & -0.013 & 0.001 & -0.226 \\
\hline S8 & -0.011 & 0.000 & -0.228 \\
\hline Mean & $\mathbf{- 0 . 0 1 3}$ & $\mathbf{0 . 0 0 0}$ & $\mathbf{- 0 . 2 2 7}$ \\
\hline STD & $\mathbf{0 . 0 0 4}$ & $\mathbf{0 . 0 0 5}$ & $\mathbf{0 . 0 0 5}$
\end{tabular}

Table 1. Lever-arm adjusted values

The Xsens MTI AHRS is assumed to have an accuracy of 0.5 degrees in roll/pitch and 1 degree in yaw. The standard deviation calculated from excentricities in $\mathrm{X}$ and $\mathrm{Y}$ (table 2) are consistent with such an accuracy. However, the standard deviation of eccentricities calculated from VO (table 3 ) has proven to be 0.1 degree lower in each direction than this provided by the AHRS. Such an accuracy is of the same level as for the EOM APX-15 (Applanix, n.d.). Both MTI and APX-15 are typical AHRS suitable for being embedded on UAV-LiDAR platforms.

\begin{tabular}{c|c|c|c} 
Station & $e_{X}[\mathrm{deg}]$ & $e_{Y}[\mathrm{deg}]$ & $e_{Z}[\mathrm{deg}]$ \\
\hline $\mathrm{S} 1$ & -1.5813 & -0.3556 & 64.1095 \\
\hline S2 & -2.0826 & 0.3589 & 81.5953 \\
\hline $\mathrm{S} 3$ & -1.4419 & 0.5784 & 85.6736 \\
\hline $\mathrm{S} 4$ & -2.3144 & 0.8517 & 79.4783 \\
\hline S5 & -2.0304 & -0.206 & 55.1928 \\
\hline S6 & -2.7594 & -1.4432 & 12.0667 \\
\hline S7 & -1.23 & -2.0044 & -11.2554 \\
\hline S 8 & -0.7299 & -1.0463 & -19.4643 \\
\hline STD & $\mathbf{- 1 . 7 7 1 2}$ & $\mathbf{- 0 . 4 0 8 3}$ & $\mathbf{4 3 . 4 2 4 6}$ \\
\hline Mean & $\mathbf{0 . 6 5 0 0}$ & $\mathbf{1 . 0 1 5 5}$ & $\mathbf{4 3 . 1 5 5 0}$
\end{tabular}

Table 2. Eccentricities adjusted values obtained for AHRS

\begin{tabular}{c|c|c|c} 
Station & $e_{X}[\mathrm{deg}]$ & $e_{Y}[\mathrm{deg}]$ & $e_{Z}[\mathrm{deg}]$ \\
\hline S1 & -88.5325 & -1.4790 & -19.4675 \\
\hline S2 & -88.5210 & -1.4904 & -19.4847 \\
\hline S3 & -88.5210 & -1.5076 & -19.4675 \\
\hline S4 & -88.4809 & -1.5019 & -19.5764 \\
\hline S5 & -88.4981 & -1.4904 & -19.6911 \\
\hline S6 & -88.4809 & -1.5019 & -19.5535 \\
\hline S7 & -88.4924 & -1.4904 & -19.5535 \\
\hline S8 & -88.5038 & -1.4675 & -19.5822 \\
\hline Mean & $\mathbf{- 8 8 . 5 0 3 8}$ & $\mathbf{- 1 . 4 9 0 4}$ & $\mathbf{- 1 9 . 5 4 7 8}$ \\
\hline STD & $\mathbf{0 . 0 1 7 2}$ & $\mathbf{0 . 0 1 1 5}$ & $\mathbf{0 . 0 7 4 5}$
\end{tabular}

Table 3. Eccentricities adjusted values obtained for Camera

\subsection{Residuals on control point coordinates}

Residuals are defined as the differences between the coordinate values of the control points obtained by the test bench measurements and the coordinate values that act as the reference. Table 4 shows the averages of residuals for each station by means of the processing that involves the AHRS data. Table 5 displays the same results but for the processing based on the images coming from the camera.

\begin{tabular}{c|c|c|c} 
Station & $\Delta_{X}[\mathrm{~m}]$ & $\Delta_{Y}[\mathrm{~m}]$ & $\Delta_{Z}[\mathrm{~m}]$ \\
\hline S1 & -0.111 & 0.406 & -0.051 \\
\hline S2 & -0.140 & 0.081 & 0.122 \\
\hline S3 & -0.144 & -0.085 & 0.124 \\
\hline S4 & -0.183 & -0.003 & 0.159 \\
\hline S5 & -0.204 & 0.087 & 0.179 \\
\hline S6 & -0.202 & 0.092 & 0.180 \\
\hline S7 & -0.187 & 0.054 & 0.166 \\
\hline S8 & -0.139 & 0.003 & 0.127 \\
\hline S9 & -0.165 & 0.068 & 0.149 \\
\hline S10 & -0.168 & 0.032 & 0.149 \\
\hline S11 & -0.189 & -0.003 & 0.165 \\
\hline S12 & -0.144 & -0.060 & 0.128 \\
\hline Mean & $\mathbf{- 0 . 1 6 5}$ & $\mathbf{0 . 0 5 6}$ & $\mathbf{0 . 1 3 3}$ \\
\hline STD & $\mathbf{0 . 0 2 9}$ & $\mathbf{0 . 1 2 4}$ & $\mathbf{0 . 0 6 2}$
\end{tabular}

Table 4. Averages of residuals for each station using the processing that involves the AHRS data.

The results indicate that the former residuals have a decimetric magnitude, whereas the latter a centimetric magnitude.

\section{CONCLUSION}

In this paper, we have presented the process we followed to assess the reliability and the accuracy of attitude extracted from 


\begin{tabular}{c|c|c|c} 
Station & $\Delta_{X}[\mathrm{~m}]$ & $\Delta_{Y}[\mathrm{~m}]$ & $\Delta_{Z}[\mathrm{~m}]$ \\
\hline S1 & -0.014 & 0.017 & -0.001 \\
\hline S2 & -0.019 & 0.018 & 0.008 \\
\hline S3 & -0.025 & 0.026 & -0.005 \\
\hline S4 & -0.011 & 0.014 & -0.007 \\
\hline S5 & -0.013 & 0.022 & -0.020 \\
\hline S6 & -0.006 & 0.021 & -0.017 \\
\hline S7 & -0.004 & 0.012 & -0.014 \\
\hline S8 & -0.015 & 0.016 & -0.016 \\
\hline S9 & -0.012 & 0.017 & -0.022 \\
\hline S10 & -0.016 & 0.016 & -0.013 \\
\hline S11 & -0.009 & 0.012 & -0.002 \\
\hline S12 & -0.038 & 0.023 & 0.0180 \\
\hline Mean & $\mathbf{- 0 . 0 1 5}$ & $\mathbf{0 . 0 1 8}$ & $\mathbf{- 0 . 0 0 8}$ \\
\hline STD & $\mathbf{0 . 0 0 9}$ & $\mathbf{0 . 0 0 4}$ & $\mathbf{0 . 0 1 2}$
\end{tabular}

Table 5. Averages of residuals for each station obtained by VO.

VO. We have built an experimental test bench with several instruments to simulate a LiDAR/camera coupled system. We have transposed the DG principle to our system including adjustment and calibration phases. Our test bench has been experimented with two scenes containing control points already known. Regarding the results presented for $\mathrm{VO}$, the residual error for the surveyed points is around 2-3 centimeters which corresponds to the accuracy level commonly attained with topographic classical surveys. The results also show that VO is more accurate than MEMS AHRS (MTI) and, at least, of the same accuracy as GNSS/INS systems (APX-15) which are generally embedded in UAV-LiDAR platforms. These findings validate conclusively the reliability of $\mathrm{VO}$ in our experiments.

However, we have not yet discuss several topics such as:

- The influence Internal Orientation Parameters (IOPs) in the adjustment phase.

- How images could be georeferenced? In our work, we have directly made use of the targets as GCPs. The next step consists in performing the same processing with only one GCP, even without, using solely the camera positions deduced from GNSS data and the canvas determined with the images.

In terms of perspective, the test bench has to be upgraded by replacing the tacheometer by a LiDAR. This requires that all the records of the different instruments, LiDAR, camera, and GNSS be time-synchronised. The final step of the project will be to embed the equipment in an UAV platform and to perform a survey in order to evaluate the accuracy of our system under real operating conditions. Besides allowing accurate direct georeferencing of LiDAR data to be performed, visual odometry provides complimentary data consisting in photogrammetry-acquired images of the surveyed scenes. The images provide us with radiometry and texture data as a complement to LIDAR-determined geometrical and dimensional data.

\section{References}

[Applanix, n.d.] Applanix, n.d. Apx-15 UAV - datasheet.

[Bäumker and Heimes, 2001] Bäumker, M. and Heimes, F., 2001. New calibration and computing method for direct georeferencing of image and scanner data using the position and angular data of an hybrid inertial navigation system. In: OEEPE Workshop, Integrated Sensor Orientation.

[Caballero et al., 2008] Caballero, F., Merino, L., Ferruz, J. and Ollero, A., 2008. Vision-based odometry and SLAM for medium and high altitude flying UAVs. In: Unmanned Aircraft Systems, Springer, pp. 137-161.

[Challis, 1995] Challis, J. H., 1995. A procedure for determining rigid body transformation parameters. Journal of biomechanics 28(6), pp. 733-737.

[Daakir et al., 2015] Daakir, M., Pierrot-Deseilligny, M., Bosser, P., Pichard, F. and Thom, C., 2015. UAV onboard photogrammetry and gps positionning for earthworks. The International Archives of Photogrammetry, Remote Sensing and Spatial Information Sciences 40(3), pp. 293.

[Deng et al., 2014] Deng, C., Wang, S., Huang, Z., Tan, Z. and Liu, J., 2014. Unmanned aerial vehicles for power line inspection: A cooperative way in platforms and communications. Journal of Communications.

[El-Sheimy, 2009] El-Sheimy, N., 2009. Emerging MEMS IMU and its impact on mapping applications. In: Photogrammetric Week, Vol. 9.

[Eling et al., 2015] Eling, C., Wieland, M., Hess, C., Klingbeil, L. and Kuhlmann, H., 2015. Development and evaluation of a UAV based mapping system for remote sensing and surveying applications. The International Archives of Photogrammetry, Remote Sensing and Spatial Information Sciences 40(1), pp. 233.

[Howard, 2008] Howard, A., 2008. Real-time stereo visual odometry for autonomous ground vehicles. In: Intelligent Robots and Systems, 2008. IROS 2008. IEEE/RSJ International Conference on, IEEE, pp. 3946-3952.

[Lin et al., 2011] Lin, Y., Hyyppa, J. and Jaakkola, A., 2011. Mini-UAV-borne LiDAR for fine-scale mapping. IEEE Geoscience and Remote Sensing Letters 8(3), pp. 426-430.

[Lowe, 2004] Lowe, D. G., 2004. Distinctive image features from scale-invariant keypoints. International journal of computer vision 60(2), pp. 91-110.

[Maimone et al., 2007] Maimone, M., Cheng, Y. and Matthies, L., 2007. Two years of visual odometry on the Mars exploration rovers. Journal of Field Robotics 24(3), pp. 169-186.

[Roussel et al., 2015] Roussel, C., Verdun, J., Cali, J., Maia, M. and d'Eu Jean-François, 2015. Integration of a strapdown gravimeter system in an autonomous underwater vehicle. In: The International Archives of the Photogrammetry, Remote Sensing and Spatial Information Sciences, Volume XL-5/W5, Underwater 3D Recording and Modeling, 16-17 April 2015, Piano di Sorrento, Italy, pp. 199-206. 
The International Archives of the Photogrammetry, Remote Sensing and Spatial Information Sciences, Volume XLII-2/W6, 2017 International Conference on Unmanned Aerial Vehicles in Geomatics, 4-7 September 2017, Bonn, Germany

[Skaloud et al., 1996] Skaloud, J., Cramer, M. and Schwarz, K., 1996. Exterior orientation by direct measurement of camera position and attitude. International Archives of Photogrammetry and Remote Sensing 31(B3), pp. 125-130.

[Wallace et al., 2012] Wallace, L., Lucieer, A., Watson, C. and Turner, D., 2012. Development of a UAV-LiDAR system with application to forest inventory. Remote Sensing 4(6), pp. 1519-1543. 